\title{
Identification of proteins suppressing the functions of oncogenic phosphatase of regenerating liver 1 and 3
}

\author{
JU-DONG LEE ${ }^{1}$, HAIYOUNG JUNG ${ }^{2}$ and SANG-HYUN MIN ${ }^{3}$ \\ ${ }^{1}$ Graduate School of Medical Science and Engineering, Biomedical Research Center, Korea Advanced Institute \\ of Science and Technology, Daejeon 305-701; ${ }^{2}$ Immunotherapy Convergence Research Center, Korea \\ Research Institute of Bioscience and Biotechnology, Daejeon 305-806; ${ }^{3}$ New Drug Development Center, \\ Daegu-Gyeongbuk Medical Innovation Foundation, Daegu 701-310, Republic of Korea
}

Received May 29, 2015; Accepted August 11, 2016

DOI: $10.3892 /$ etm.2016.3722

\begin{abstract}
The phosphatase of regenerating liver (PRL) family, including PRL-1, PRL-2, and PRL-3, comprises protein tyrosine phosphatases whose deregulation is associated with the tumorigenesis and metastasis of many types of cancer. However, the underlying mechanism is poorly understood. In this study, aiming to increase understanding of the molecular mechanisms underlying the functions of PRL-1 and PRL-3, a yeast two-hybrid system was employed to screen for their interacting proteins. Alignment with the NCBI BLAST database revealed 12 interactive proteins: Synaptic nuclear envelope protein 2, emerin, mannose 6-phosphate receptor-binding protein 1 , low-density lipoprotein receptor-related protein 10 , Rab acceptor 1, tumor protein D52-like 2, selectin P ligand (SELPLG), guanylate binding protein 1, transmembrane and ubiquitin-like domain-containing 2, NADH:ubiquinone oxidoreductase subunit B8, syndecan 4 and FK506-binding protein 8 (FKBP8). These proteins are associated with cell proliferation, apoptosis, immune response, cell fate specification and metabolic process in biological process categories, and involved in various signaling pathways, including Alzheimer's disease, Parkinson's disease, Huntington's disease, hypertrophic cardiomyopathy and cell adhesion molecules. Interactions of PRL-1 with the prey proteins SELPLG and FKBP8 were confirmed by immunoprecipitation or immunostaining. Furthermore, SELPLG and FKBP8 suppressed PRL-1- or PRL-3-mediated p53 activity. Identification of the proteins interacting with PRL family proteins may provide valuable information to better
\end{abstract}

Correspondence to: Dr Sang-Hyun Min, New Drug Development Center, Daegu-Gyeongbuk Medical Innovation Foundation, 80 Cheombok-ro, Dong-gu, Daegu 701-310, Republic of Korea E-mail: shmin03@dgmif.re.kr

Key words: phosphatase of regenerating liver 1, phosphatase of regenerating liver 3, screening, yeast two-hybrid, SYNE2, EMD, PLIN3, LRP10, RABAC1, TPD52L2, SELPLG, GBP1, TMUB2, NDUFB8, SDC4, FKBP8. understand the mechanism of PRL-mediated signal transduction in cancer and other diverse diseases.

\section{Introduction}

Phosphatases of the regenerating liver [PRLs; also known as the protein tyrosine phosphatase type IVA (PTP4A) family] were originally identified as immediate-early genes in the regenerating liver (1). The PRL family is a group of protein tyrosine phosphatases (PTPs) and plays a role in the development and metastasis of various cancers, including colorectal, prostate, breast, gastric and liver cancers, and particularly in metastatic cancers $(2,3)$. The PRL family comprises three genes: PRL-1, PRL-2 and PRL-3. The overexpression of the PRL family has been frequently reported in various cancers, especially in metastatic cancers (4-8). Overexpression of PRLs in normal cells has been found to promote proliferation, migration, and invasion $(4,8,9)$ whereas the reduction of PRLs in cancer cells using small interfering RNA (siRNA) has been shown to inhibit cell motility and metastatic characteristics in a mouse model (10).

PRLs affect a number of signaling pathways associated with cell growth and cancer development. During tumorigenesis, PRLs have been found to modulate integrin $\beta 1$-extracellular signal-regulated kinase $1 / 2$, phosphoinositide 3-kinase/AKT, keratin 8, C-terminal Src kinase, Rho GTPase, cyclin-dependent kinase 2, p53 and FK506-binding protein 8 (FKBP8) signaling pathways $(9,11-19)$.

Although it is important to elucidate the role of PRLs in cancer progression and the signaling pathways they affect, a major challenge to the analysis of the detailed signaling mechanism of PRLs is the lack of a physiologically relevant substrate and knowledge of its regulation by physical interaction. Several PRL-interacting proteins such as activating transcription factor-7, $\beta$-subunit of geranylgeranyl transferase-II, cadherin 22, ezrin, elongation factor 2 , keratin 8 , integrin- $\alpha 1$, PRL-1 (trimer), PRL-3 (oligomer) and FKBP8 have been reported $(1,11,16,20-27)$.

PRL family members have been identified to be useful biomarkers and therapeutic targets in cancer as well as in metastatic cancer due to the aforementioned properties $(1,3,27)$. However, little is known about the proteins that bind to PRL 
and regulate PRL function or are regulated by PRL. Therefore, in the present study, to screen for novel PRL-interacting proteins, yeast two-hybrid methodology was applied using PRL-1 and PRL-3 as bait. The identification of PRL-binding proteins may be useful in providing a novel insight into the mechanisms of tumorigenesis and other diseases, and might eventually lead to the development of more effective therapies.

\section{Materials and methods}

Cell culture, plasmid and reagents. HEK293T, HeLa and U2OS cell lines (American Type Culture Collection, Manassas, VA, USA) were cultured under an atmosphere of $5 \% \mathrm{CO}_{2}$ at $37^{\circ} \mathrm{C}$ in Dulbecco's modified Eagle's medium (Hyclone; GE Healthcare Life Sciences, Logan, UT, USA) supplemented with $10 \%$ fetal bovine serum (Hyclone; GE Healthcare Life Sciences), $100 \mathrm{IU} / \mathrm{ml}$ penicillin, and $100 \mu \mathrm{g} / \mathrm{ml}$ streptomycin (Gibco-BRL; Thermo Fisher Scientific, Inc., Waltham, MA, USA). cOmplete ${ }^{\mathrm{TM}}$ Mini Protease Inhibitor Cocktail tablets and Phosphatase Inhibitor Cocktail tablets were obtained from Roche Applied Science (Penzberg, Germany). Antibody against high availability (HA) probe was purchased from Santa Cruz Biotechnology, Inc. (Dallas, TX, USA; cat. no. SC-805) and antibodies against Flag ${ }^{\circledR}$ M2 (cat. no. F3165) and $\beta$-actin (cat. no. A5441) were purchased from Sigma-Aldrich (Merck Millipore, Darmstadt, Germany).

Flag-PRL-1 and Flag-PRL-3 $(12,19,28)$ were digested with restriction enzymes (EcoRI/XhoI) and cloned into the yeast expression vector pLexA (Clontech Laboratories, Inc., Mountainview, CA, USA) to form pLexA-PRL-1 and pLexA-PRL-3, respectively. The authenticity and correct orientation of the cloned sequence were then confirmed by restriction digestion and polymerase chain reaction (PCR).

Two cDNA clones encoding FKBP8 and SELPLG from pJG4-5 (Clontech Laboratories, Inc.) were inserted into a pcHA vector (Addgene vector database) to express their proteins in mammalian cells. Prey genes were digested with restriction enzymes (EcoRI/XhoI) and cloned into the mammalian expression vector pcHA. Insertion of the prey genes were confirmed by restriction enzyme digestion and nucleotide sequencing.

PCR. The DNA used for the PCR was obtained from bacterial plasmid DNA (Bioneer Corporation, Daejeon, Korea). PCR was performed with the following primer pairs: PRL-1 forward, 5'-TACACACAATCCAACCAATG-3', and reverse, 5'-AATTAATGCTAGGGCAACAA-3', and PRL-3 forward, 5'-TCATTGAGGACCTGAAGAAG-3', and reverse, 5'-CTCAGCCAGTCTTCCACTAC-3'. PCR pre-mix was used for the reaction (Bioneer Corporation). In each reaction, $20 \mu \mathrm{l}$ final reaction mixture contained $10 \mu$ l Premix Taq, $0.8 \mathrm{ml}$ PCR forward primer $(10 \mathrm{~mm}), 0.8 \mathrm{ml}$ reverse primer $(10 \mathrm{~mm}), 2 \mu \mathrm{l}$ DNA $(100 \mathrm{ng} / \mu \mathrm{l})$ and $\mathrm{dH}_{2} \mathrm{O}$. Subsequently, the reaction mixture was incubated at $95^{\circ} \mathrm{C}$ for $5 \mathrm{~min}$, followed by 40 cycles of $95^{\circ} \mathrm{C}$ for $15 \mathrm{sec}$ and $60^{\circ} \mathrm{C}$ for $45 \mathrm{sec}$ with 20 cycles. $1.5 \%$ agarose gel was used for electrophoresis of the PCR product.

Screening of a HeLa library and selection of proteins interacting with PRL-1 and PRL-3. The cDNA from a HeLa library (Clontech Laboratories, Inc.) was sub-cloned into pJG4-5 vectors (Clontech Laboratories, Inc.) for yeast two-hybrid screening. The EGY48 yeast strain (Clontech Laboratories, Inc.) was transformed with pLexA-PRL-1 or pLexA-PRL-3 by a small-scale yeast transformation protocol (28) and plated onto synthetic defined (SD)/-Trp1 (without yeast gene Trp1) medium (Sigma-Aldrich) and grown at $30^{\circ} \mathrm{C}$ for $2-4$ days. Selected clones were grown in $2 \mathrm{ml}$ yeast extract peptone dextrose medium containing ampicillin at $30^{\circ} \mathrm{C}$ overnight with shaking. The yeast strain expressing LexA-PRL-1 or PRL-3 bait protein was transformed with the HeLa cDNA library fused to the GAL-4 activation domain by the lithium acetate method (large-scale yeast transformation protocol) (28). The cDNA library was screened using a yeast two-hybrid system (Matchmaker LexA two-hybrid system; Clontech Laboratories, Inc.) to detect interacting proteins, according to the manufacturer's protocol. Positive clones were selected and assayed for lacZ reporter activity using a filter $\beta$-galactosidase assay with X-Gal. Plasmids from positive yeast clones were isolated and transformed into competent cells. Plasmids isolated from competent cells were transformed into XL1-blue competent cells (Agilent Technologies, Inc.- Santa Clara, CA, USA) for analysis of the insert size and for sequencing. The interaction between LexA-PRl-1 or PRL-3 and positive clones was confirmed by small-scale yeast transformation.

DNA sequences were determined (Bioneer Corporation) and nucleotide sequence databases were searched for homologous sequences by Basic Local Alignment Search Tool (BLAST) analysis (https://blast.ncbi.nlm.nih.gov/Blast.cgi).

Transfection, immunoprecipitation and immunoblot analysis. PRL-1 or PRL-3 expression vectors were transfected into each cell line (HEK293T, HeLa and U2OS) using Lipofectamine Plus (Gibco-BRL; Thermo Fisher Scientific, Inc.), using the manufacturer's protocol. After $48 \mathrm{~h}$, the cells were washed and lysed with lysis buffer containing $150 \mathrm{mM} \mathrm{NaCl}, 0.1 \%$ Nonidet P-40 and 50 mM Tris- $\mathrm{Cl}$ (pH 7.4). Detergent-insoluble materials were removed via centrifugation $(1,000 \times \mathrm{g})$, and the clear lysates were incubated with anti-Flag ${ }^{\circledR}$ M2 antibody (1:500) and Protein G Plus Agarose beads for $4 \mathrm{~h}$ (Santa Cruz Biotechnology, Inc.). The beads were washed three times with lysis buffer (29). For immunoblotting, coprecipitates or whole cell extracts were resolved via $10 \%$ sodium dodecyl sulfate-polyacrylamide gel electrophoresis, and subsequently transferred to nitrocellulose membranes. The membranes were immunoblotted with anti-HA $(1: 10,000)$ and anti-Flag ${ }^{\circledR}$ M2 (1:2,000) antibodies and then developed with an enhanced chemiluminescence detection system (Thermo Fisher Scientific, Inc.).

Immunofluorescence analysis. U2OS cells $(50,000)$ were plated on coverslips pretreated with $0.1 \%$ gelatin in 12 -well dishes, then transfected with indicated expression vectors (HA-SELPLG, HA-FKBP8 and/or Flag-PRL-1) and incubated for 2 days. The transfected cells were washed with phosphate-buffered saline (PBS), fixed for $20 \mathrm{~min}$ in $4 \%$ (w/v) paraformaldehyde, permeabilized for $10 \mathrm{~min}$ at room temperature with PBS containing $0.3 \%$ (v/v) Triton X-100, and further incubated for $10 \mathrm{~min}$ in $1 \%$ bovine serum albumin (Sigma-Aldrich). Samples were subsequently incubated for $1 \mathrm{~h}$ with primary antibodies anti-HA $(1: 10,000)$ and anti-Flag ${ }^{\circledR}$ M2 
$(1: 2,000)$, washed three times with PBS, and then incubated with Alexa Fluor 488-conjugated goat antibody against mouse IgG and Alexa Fluor 594-conjugated goat antibody against rabbit IgG (Molecular Probes; Thermo Fisher Scientific, Inc.). The coverslips were mounted on glass slides in Vectashield medium (Vector Laboratories, Inc., Burlingame, CA, USA). Images were acquired using a Leica 6000 microscope (Leica Microsystems, Inc., Buffalo Grove, IL, USA). For DAPI staining, $1 \mathrm{ml}$ DAPI $(3 \mu \mathrm{M})$ in staining buffer $(100 \mathrm{mM}$ Tris, $\mathrm{pH} 7.4,150 \mathrm{mM} \mathrm{NaCl}, 1 \mathrm{mM} \mathrm{CaCl}, 0.5 \mathrm{mM} \mathrm{MgCl}_{2}, 0.1 \%$ Nonidet P-40) was added to each cell sample and incubated for $15 \mathrm{~min}$ at room temperature.

Dual-luciferase assay. HeLa cells were transfected with pRGC-luc (28), along with each expression vector (HA-SELPLG, HA-FKBP8, Flag-PRL-1 and/or Flag-PRL-3) as indicated using Lipofectamine Plus. The cells were lysed, and the luciferase activity was evaluated using a dual luciferase assay kit (Promega Corporation, Madison, WI, USA). The data were normalized to the expression levels of a cotransfected Renilla luciferase activity reporter control.

Functional classification, pathway analysis and protein interaction network. The 12 identified proteins were sorted by pathway and the Gene Ontology (GO) categories using the DAVID database. SELPLG was selected in the Biocarta pathway. For the network of the PRL-1, PRL-3 and prey proteins, the cellular protein interaction network was constructed based on the screened proteins in this study and in the STRING database.

\section{Results}

Screening of interacting proteins with PRL-1 or 3 using a yeast two-hybrid system. The PRL family plays a significant role in the development and cancer metastasis, and shares a high degree of sequence similarity. Notably, PRL-3 has $>75 \%$ amino-acid sequence similarity to PRL-1, with a conserved function $(1,27,30)$.

To screen novel PRL-interacting proteins, human PRL-1 and PRL-3 were used as bait in a yeast two-hybrid system. Flag-PRL-1 and Flag-PRL-3 were digested with restriction enzymes $(E c o \mathrm{RI} / \mathrm{X} h \mathrm{I})$ and the inserts were cloned into the yeast expression vector pLexA (Fig. 1A). To confirm the cloning, PCR products of full length PRL-1 and PRL-3 from pLexA-PRL-1 and pLexA-PRL-3 were identified by nucleotide electrophoresis (data not shown). In addition, the inserts of PRL-1 and PRL-3 from pLexA-PRL-1 and pLexA-PRL-3 were investigated by nucleotide electrophoresis following digestion with same restriction enzymes (Fig. 1B). Also, the sequence and the orientation of the inserts were confirmed by sequencing analysis. Finally, the expression of the PRL-1 bait in yeast EGY48 was confirmed by western blotting (Fig. 1C).

A HeLa cDNA library was transformed in yeast EGY48 strains transformed with pLexA-PRL-1 or pLexA-PRL-3 bait vector expressing PRL-1 or PRL-3 and cultured at $30^{\circ} \mathrm{C}$ for $2-4$ days until colonies appeared. Finally 38 blue colonies were observed on SD/-His/-Leu/-Trp/X-Gal plates, the colonies were inoculated in SD/-Leu/-Trp liquid medium and the plasmids were extracted. Purified plasmids were retransformed in yeast EGY48 strains containing pLexA-PRL-1 or PRL-3 bait vector and blue colonies were observed again on SD/-His/-Leu/-Trp/X-Gal plates (data not shown). Plasmids isolated from yeast were transformed into XL1-blue competent cells for further analysis of the insert size and for sequencing. Inserted fragments of library plasmids were mostly between 500 and 2,000 bp in size. Identity of the prey was determined by performing BLAST search analysis. The results of the BLAST search against the human gene database indicated that 12 genes interact with PRL-1 or PRL-3: Synaptic nuclear envelope protein 2 (SYNE2), emerin (EMD), mannose 6-phosphate receptor-binding protein 1 (perilipin 3; PLIN3), low-density lipoprotein receptor-related protein 10 (LRP10), Rab acceptor 1 (RABAC1), tumor protein D52-like 2 (TPD52L2), selectin P ligand (SELPLG), guanylate binding protein 1 (GBP1), transmembrane and ubiquitin-like domain-containing 2 (TMUB2), NADH:ubiquinone oxidoreductase subunit B8 (NDUFB8), syndecan 4 (SDC4) and FKBP8 (Table I) were identified. Among them, 9 prey proteins were isolated from screening using PRL-1 bait and 6 prey proteins were obtained from screening using PRL-3 bait. There were 3 prey proteins, namely NDUFB8, FKBP8 and SDC4, that were identified from both PRL-1 and PRL-3 baits (Fig. 1D).

In vivo binding and colocalization. From among the 12 candidate genes interacting with PRL-1 or PRL-3, two cDNA clones encoding for FKBP8 and SELPLG were inserted into pcHA vector to express their proteins in mammalian cells. Prey genes were digested with restriction enzymes (EcoRI/XhoI) and cloned into the mammalian expression vector pcHA. Insertion of the prey genes was confirmed by restriction enzyme digestion and nucleotide sequencing (Fig. 1E).

To confirm their binding in a yeast-independent interaction assay, coimmunoprecipitation experiments were performed. HEK293T cells were co-transfected with Flag-PRL-1 and HA-FKBP8 or HA-SELPLG constructs, and cell extracts were then subjected to immunoprecipitation with anti-Flag antibody, followed by immunoblotting analysis with an anti-HA antibody. HA-tagged FKBP8 and SELPLG were detected in anti-Flag-PRL-1 immunoprecipitates (Fig. 2A).

The localization of bait proteins and prey proteins was then examined. U2OS cells were transfected with Flag-PRL-1, and HA-FKBP8 or HA-SELPLG. Localization of FLAG tagged-PRL-1 was visualized with anti-FLAG primary antibody and Fluor 488-conjugated goat antibody against mouse IgG and localization of HA-tagged preys was visualized with anti-HA antibody and Alexa Fluor 594-conjugated goat antibody against rabbit IgG.

In cells, PRLs are typically associated with the plasma membrane and early endosome $(1,27,30)$. An important mechanism responsible for this localization is prenylation, a post-translational lipid modification that commonly targets proteins to membranes $(3,27,30)$. Fig. $2 \mathrm{~B}$ and Table II show that PRL-1 localization is observed in the endosome, early endosome, endoplasmic reticulum, spindle, cytoskeleton, plasma membrane, microtubule cy toskeleton and intracellular non-membrane-bounded organelle. SELPLG is visible in the membrane fraction, insoluble fraction, plasma membrane, and is integral to the plasma membrane while FKBP38 
A
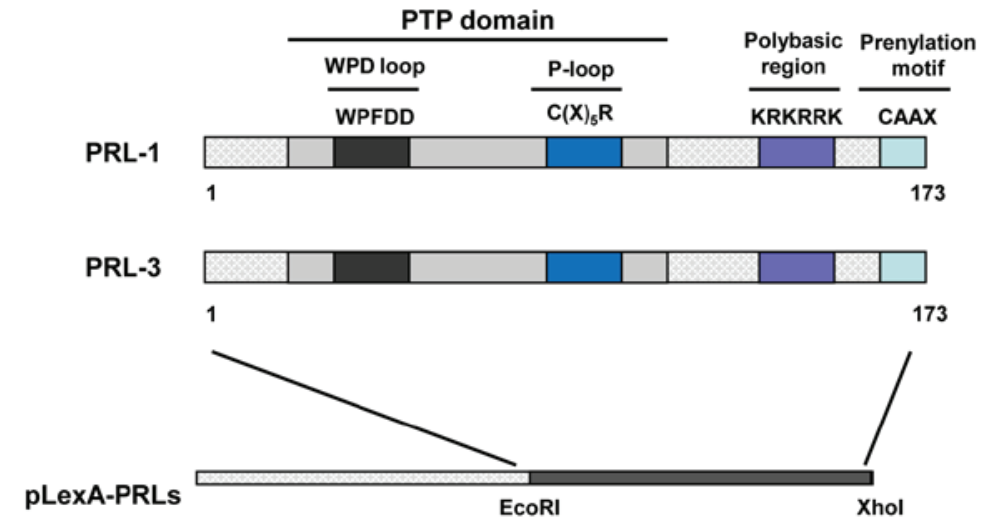

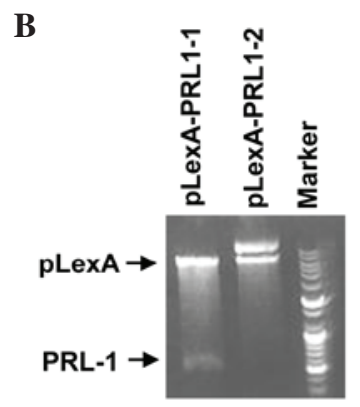

D

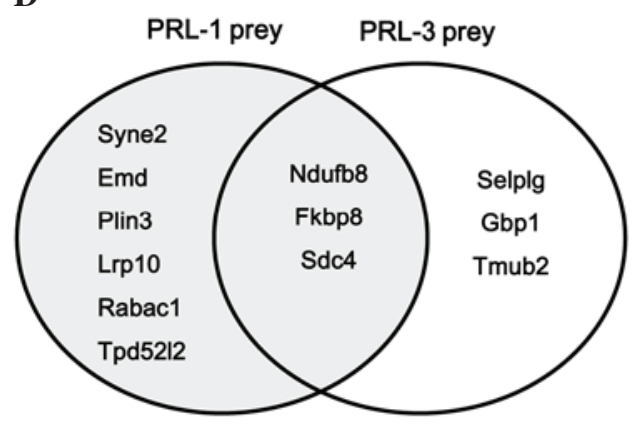

C

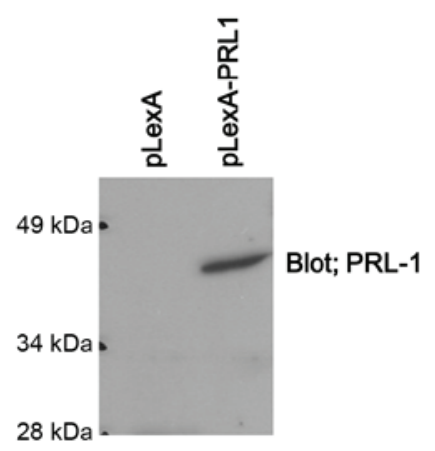

$\mathbf{E}$

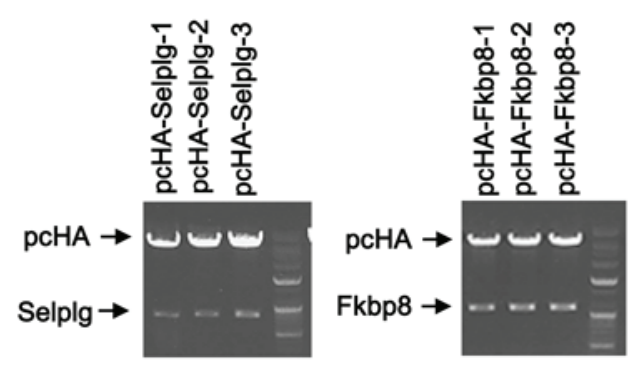

Figure 1. Screening of interacting proteins with PRL-1 or -3 using a yeast two-hybrid system. (A) Schematic representation of the PRL-1 and PRL-3 cloning strategy for the yeast two-hybrid assay. Full length PRL-1 and -3, containing a PTP domain, polybasic region and prenylation motif were inserted into pLexA bait vector. (B) Construction and identification of the bait vectors. Gel electrophoresis images of the vector (pLexA) and bait genes (PRL-1 and PRL-3). The plasmids were digested with EcoRI and XhoI, and samples were loaded onto agarose gel. (C) Expression of bait fusion protein in yeast. EGY48 yeast strain transformed with pLexA-PRL-1 or empty vector (pLexA) as a control were lysed and resolved using sodium dodecyl sulfate-polyacrylamide gel electrophoresis for western blotting using anti-PRL-1 antibody. (D) Diagram of screening results and cloning of prey genes in mammalian expression vector. Twelve prey genes were identified from screening; 9 genes were isolated from screening using PRL-1 bait and 6 genes were isolated from PRL-3 bait screening. (E) Selplg and Fkbp8 prey cDNAs were inserted into pcHA vector for mammalian expression and digested with EcoRI and XhoI restriction enzymes. PRL, phosphatase of regenerative liver. PTP, protein tyrosine phosphatase.

is observed in the mitochondrial envelope, endoplasmic reticulum membrane, plasma membrane, endomembrane system and nuclear envelope-endoplasmic reticulum network (Fig. 2B and Table II). The expression of SELPLG and FKBP38 appears to be partially colocalized with PRL-1. In the presence of preys, changes in the localization of PRL-1 were not observed, suggesting that the expression of these preys does not affect the prenylation and localization of PRL-1.

SELPLG and FKBP8 inhibit the functions of PRL-1 and $P R L-3$. Having verified the binding of FKBP8 and SELPLG with PRL-3 protein, the next important question is whether
FKBP8 and SELPLG affect the functions of PRL-1 and PRL-3 in cells. The roles of PRL-1 and PRL-3 are associated with the downregulation of p21 transcription as well as the activity of p53 (28). Therefore, the effects of two prey proteins on the downregulation of $\mathrm{p} 53$ reporter activities mediated by PRL-1 and PRL-3 were investigated. HeLa cells were transfected with each prey protein and/or Flag-PRL-1 (or Myc-PRL-3) and p53-luciferase reporter (pRGC-luc) (Fig. 3). When p53-luc was transfected with PRL-1 or PRL-3, inhibition of luciferase activity was observed (Fig. 3A), as shown previously (28). However, SELPLG and FKBP8 markedly attenuated the PLR-1-mediated p53-luc inhibition (Fig. 3A). Also, similar results were observed when SELPLG 
A

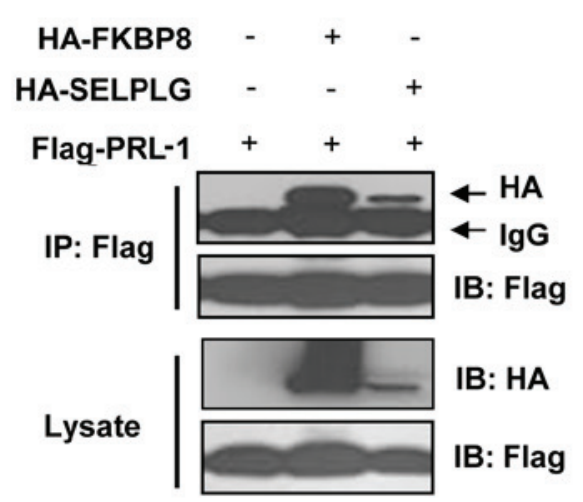

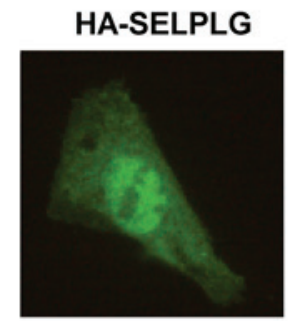

HA-FKBP8

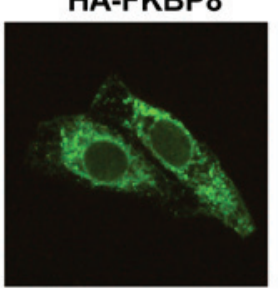

Flag-PRL-1

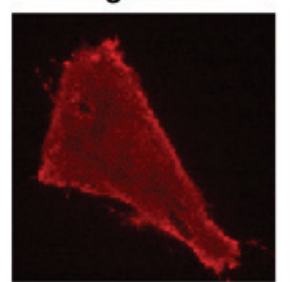

Flag-PRL-1

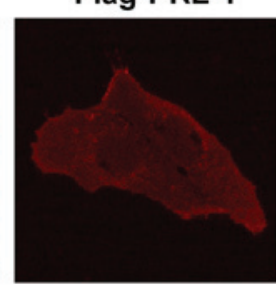

DAPI

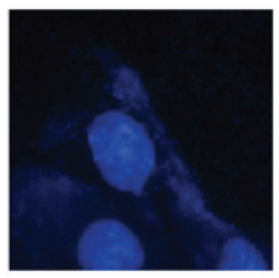

DAPI

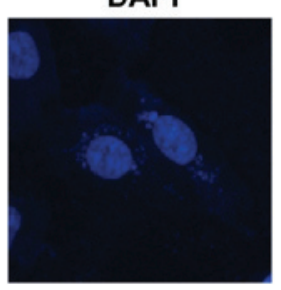

Merge

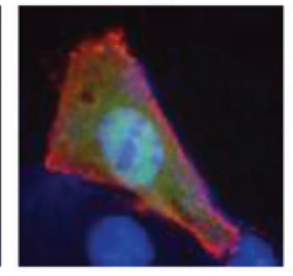

Merge

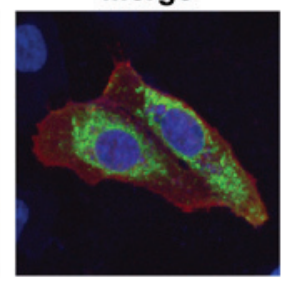

Figure 2. In vivo binding and colocalization. (A) FKBP8 and SELPLG interact with PRL-1. Flag-PRL-1 and/or HA-FKBP8 or HA-SELPLG were transfected into HEK293T cells. The cells were treated with MG132 for $4 \mathrm{~h}$ prior to harvesting, and $48 \mathrm{~h}$ later, the cells were prepared for co-IP and western blot analysis. (B) Colocalization of FKBP8 or SELPLG with PRL-1. Flag-PRL-1 and HA-FKBP8 or HA-SELPLG were transfected in U2OS cells. Then, $48 \mathrm{~h}$ later, the cells were prepared for immunofluorescence analysis. Images were acquired using a Leica 6000 microscope (magnification, x200). FKBP8, FK506-binding protein 8; SELPLG, selectin P ligand; PRL-1, phosphatase of regenerating liver 1; HA, high availability; DAPI, 4',6-diamidino-2-phenylindole; IP, immunoprecipitation; IB, immunoblotting.

and FKBP8 were introduced with PRL-3 (Fig. 3B). These findings reveal that SELPLG and FKBP8 inhibit the ability of PRL-1 and PRL-3 to reduce p53 reporter activity and imply that SELPLG and FKBP8 inhibit the cellular functions of PRL-1 and PRL-3.

Functional classification, pathway analysis and protein interaction network. The identified proteins were sorted according to pathways and GO categories using the DAVID bioinformatics resource. Pathways for SELPLG were identified using the BioCarta pathway database (data not shown). Pathways for NDUFB8, EMD, SELPLG and SDC4 were identified using KEGG pathway analysis and contained oxidative phosphorylation, Alzheimer's disease, Parkinson's disease, Huntington's disease, hypertrophic cardiomyopathy, arrhythmogenic right ventricular cardiomyopathy, dilated cardiomyopathy, cell adhesion molecules, adhesion and diapedesis of granulocytes, cells and molecules involved in local acute inflammatory response, and extracellular matrix (ECM)-receptor interaction (Table III). Among the 12 proteins, there were 9 proteins involved in diverse biological processes including vesicle transport, protein folding, cell proliferation, apoptosis, immune response, cell fate specification and metabolic process (Table IV). Cellular component data showed that the localizations of the 12 proteins mostly or partly matched with those of PRL-1 or PRL-3 (Table II).

A PRL-1 and PRL-3-prey proteins interaction network was constructed using the STRING database (Fig. 4). SDC4, PLIN3, SYNE2, TPD52L2, EMD and FKBP8 were indicated to by the most closely-related and specific node proteins associated with PRL-3, whereas SELPLG, GBP1, RABAC1 and NDUFB8 were the most remarkable node proteins associated with PRL-1. TMUB2 and LRP10 did not show any indirect interactions with PRL-1 or PRL-3 (Fig. 4). These notable node proteins appear to be particularly important in the regulation and organization of PRL-1 and PRL-3 in the prey proteins interaction network.

\section{Discussion}

The PRL family comprises a group of PTPs that play an important role in the development and metastasis of various types of cancer (12). The family members, which include PRL-1, PRL-2 and PRL-3, share a high degree of sequence similarity and show similar functional characteristics. It has been reported that several signaling pathways involved in cell growth and cancer development are affected (regulated by) PRLs $(3,4)$. However, the mechanisms by which PRLs regulate signaling or interact with direct binding partners to mediate their effects remains to be clearly elucidated.

In the present study, 12 proteins interacting with PRL-1 or PRL-3 were identified using a yeast two-hybrid system. GO biological process data indicated that these proteins are mostly associated with nuclear envelope organization, endomembrane organization and nucleus organization (Table IV). Cellular components data suggest that they are located at membrane parts, integral to membrane, intrinsic to membrane, envelope, nuclear membrane, contractile fiber part, myofibril, organelle membrane and nuclear envelope (Table II). Molecular functions of 6 genes were classified as protein binding (data not shown). They were also found to be involved in various signaling pathways such as oxidative phosphorylation, Alzheimer's disease, hypertrophic cardiomyopathy, ECM-receptor interaction and cell adhesion molecules in KEGG pathways (Table III).

FKBP8 is a member of the FKBP family of proteins, and is widely expressed in cancer cell lines $(31,32)$. In cancer, FKBP8 has potential antitumor effects via the regulation of anti-invasive syndecan 1 , proinvasive matrix metalloproteinase $9(33,34)$, mechanistic target of rapamycin, Rheb-GTP (35) and PRL-3 (28). Results of our previous study showed that FKBP8 
Table I. List of the identified preys from screening.

\begin{tabular}{clll}
\hline Prey no. & Bait & Symbol & \multicolumn{1}{c}{ Full name } \\
\hline 1 & PRL-1 & SYNE2 & Synaptic nuclear envelope protein 2 \\
2 & PRL-1 & EMD & Emerin \\
3 & PRL-1 & PLIN3 & Mannose 6-phosphate receptor-binding protein 1 \\
4 & PRL-1 & LRP10 & Low-density lipoprotein receptor-related protein 10 \\
5 & PRL-1 & RABAC1 & Rab acceptor 1 \\
6 & PRL-1 & TPD52L2 & Tumor protein D52-like 2 \\
7 & PRL-3 & SELPLG & Selectin P ligand \\
8 & PRL-3 & GBP1 & Guanylate binding protein 1 \\
9 & PRL-3 & TMUB2 & Transmembrane and ubiquitin-like domain-containing 2 \\
10 & PRL-1, PRL-3 & NDUFB8 & NADH:ubiquinone oxidoreductase subunit B8 \\
11 & PRL-1, PRL-3 & FKBP8 & FK506-binding protein 8 \\
12 & PRL-1, PRL-3 & SDC4 & Syndecan 4 \\
\hline
\end{tabular}

PRL, phosphatase of regenerating liver.

Table II. Analysis of the cellular components associated with the identified proteins, based on the cellular components gene ontology categories of DAVID.

Gene

Cellular components

FKBP8

Mitochondrial envelope, endoplasmic reticulum membrane, plasma membrane, nuclear envelope-endoplasmic reticulum network

NDUFB8

Mitochondrion, mitochondrial envelope, endoplasmic reticulum, integral to membrane, NADH dehydrogenase complex

RABAC1

Golgi apparatus, plasma membrane, synaptic vesicle, integral to membrane, cell junction, membrane-bounded vesicle, synapse

EMD

Nuclear envelope, endoplasmic reticulum, spindle, cytoskeleton, endomembrane system, microtubule cytoskeleton, nuclear membrane

GBP1

LRP10

Plasma membrane, internal side of plasma membrane, plasma membrane part

PLIN3

Coated pit, endomembrane system, integral to membrane, intrinsic to membrane

Endosome, Golgi apparatus, lipid particle, plasma membrane, internal side of plasma membrane, monolayer-surrounded lipid storage body

SELPLG

Cell fraction, membrane fraction, insoluble fraction, plasma membrane, intrinsic to plasma membrane

SYNE2 Nuclear envelope, cytoskeleton, plasma membrane, endomembrane system, integral to membrane, nuclear membrane

SDC4 Golgi apparatus, plasma membrane, adherens junction, focal adhesion, cell surface, cellsubstrate junction, membrane raft, anchoring junction

TMUB2 Integral to membrane, intrinsic to membrane

TPD52L2 Perinuclear region of cytoplasm

PRL-1 Endosome, endoplasmic reticulum, spindle, cytoskeleton, plasma membrane, microtubule cytoskeleton

PRL-3

Endosome, early endosome, plasma membrane

DAVID, Database for. Annotation, Visualization, and Integrated Discovery.

binds to PRL-3, and suppresses PRL-3-mediated p53 activity and cell proliferation (28). The present study also provided evidence that FKBP8 binds to PRL-1, and suppresses the function of PRL-1, in addition to that of PRL-3.
SELPLG is a glycoprotein that acts as a counter-receptor for the cell adhesion molecules P-, E- and L-selectin expressed on myeloid cells and T lymphocytes (36). In leukocyte trafficking during inflammation, SELPLG tethers leukocytes 
Table III. Signal pathway analysis of the identified proteins, based on the pathway categories of DAVID.

\begin{tabular}{ll}
\hline Gene & \multicolumn{1}{c}{ Signaling pathway } \\
\hline NDUFB8 & Oxidative phosphorylation, Alzheimer's disease, Parkinson's disease, Huntington's disease \\
EMD & Hypertrophic cardiomyopathy, arrhythmogenic right ventricular cardiomyopathy, dilated cardiomyopathy \\
SELPLG & Cell adhesion molecules, adhesion and diapedesis of granulocytes, cells and molecules involved in local \\
& acute inflammatory response \\
SDC4 & ECM-receptor interaction, cell adhesion molecules
\end{tabular}

DAVID, Database for. Annotation, Visualization, and Integrated Discovery; ECM, extracellular matrix.

Table IV. Biological process analysis of the identified proteins, based on the biological process gene ontology categories of DAVID.

Gene Biological process

FKBP8

Cell fate specification, regionalization, protein folding, apoptosis, smoothened signaling pathway, pattern specification process, dorsal/ventral pattern formation, neural tube patterning and development, regulation of BMP signaling pathway, chordate embryonic development

NDUFB8 Oxidative phosphorylation, mitochondrial electron transport, NADH to ubiquinone, phosphorus metabolic process, energy derivation by oxidation of organic compounds, phosphorylation, cellular respiration, oxidation reduction

EMD Muscle system process, muscle contraction, nucleus organization, nuclear envelope organization, muscle organ development, endomembrane organization, membrane organization, nuclear envelope reassembly

GBP1 Immune response

LRP10 Lipid transport, endocytosis, membrane invagination, lipid localization, membrane organization, vesicle-mediated transport

PLIN3 Vesicle-mediated transport

SELPLG Cell motion, leukocyte adhesion, cell-cell adhesion, cell migration, biological adhesion, cellular extravasation, cell motility, leukocyte migration, leukocyte tethering or rolling, localization of cell

SDC4 Regulation of muscle contraction, regulation of phosphate metabolic process, regulation of phosphorylation, positive regulation of catalytic activity, regulation of kinase activity, regulation of system process, regulation of molecular function, regulation of transferase activity

TPD52L2 Regulation of cell proliferation

PRL-1 Protein amino acid dephosphorylation, phosphate metabolic process, cell cycle, regulation of cell migration, regulation of locomotion, regulation of cell motion

PRL-3 Protein amino acid dephosphorylation, phosphorus metabolic process, phosphate metabolic process

DAVID, Database for. Annotation, Visualization, and Integrated Discovery; BMP, bone morphogenetic protein.

to activating platelets or selectin-expressing endothelia. SELPLG requires post-translational modification by tyrosine sulfation and addition of the sialyl-Lewis-x tetrasaccharide for its high-affinity binding activity. Aberrant expression of and polymorphisms in the SELPLG gene are associated with defects in the innate and adaptive immune response.

In the present study, 12 potential PRL-1/3 binding proteins were identified, including 11 novel binding partners and a known binding partner, FKBP8. SELPLG and FKBP8 proteins were shown to directly bind to PRL-1 and inhibit the downregulation of p53 reporter activities mediated by PRL-3 and PRL-1. These results demonstrate that SELPLG and FKBP8 may be regulators of the oncogenic proteins PRL-1 and PRL-3 and can have a marked impact on cell proliferation.
It is possible that the 12 PRL-binding proteins positively or negatively regulate PRL function (FKBP8 and SELPLG) or may be regulated by PRLs. In regard to this hypothesis, further studies are underway to reveal those mechanisms.

In conclusion, multiple PRLs binding proteins were screened using a yeast two-hybrid system. The identified proteins are associated with diseases including Alzheimer's disease, Parkinson's disease, Huntington's disease, hypertrophic cardiomyopathy, arrhythmogenic right ventricular cardiomyopathy and dilated cardiomyopathy, suggesting that the PRL family may be involved in diverse diseases as well as cancer. Furthermore, these findings may provide valuable information for better understanding the interactions between the PRL family and target proteins, and revealing new biological functions of PRLs. 
A

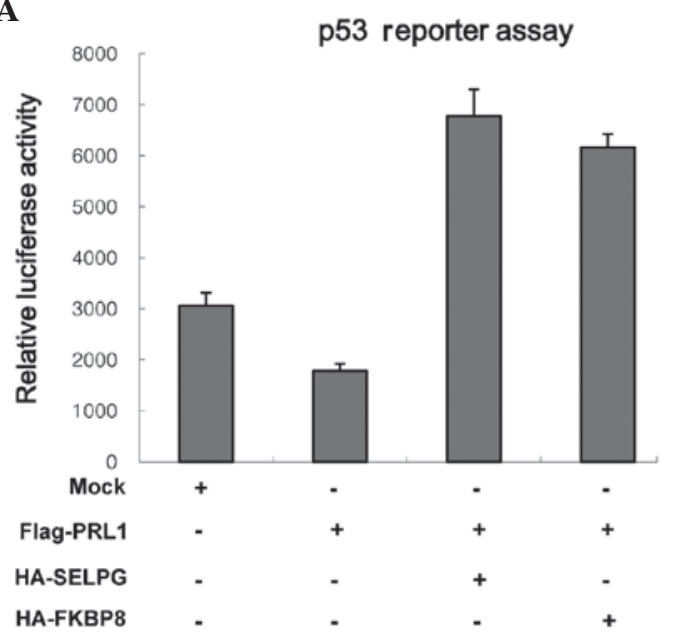

B

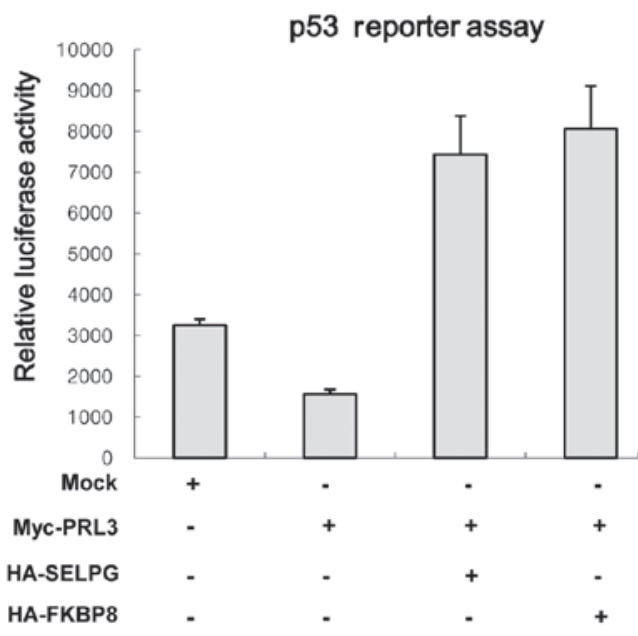

Figure 3. SELPLG and FKBP8 suppress the function of PRL-1 and -3. (A) The effect of PRL-1 on the p53 reporter is attenuated by SELPLG and FKBP8. The p53 reporter vector (100 ng) was transfected with PRL-1 (200 ng) plus HA-FKBP8 or HA-SELPLG (0 or $400 \mathrm{ng})$ as indicated. (B) The effect of PRL-3 on the p53 reporter is recovered by SELPLG and FKBP8. The p53 reporter vector (100 ng) was transfected with PRL-3 (200 ng) plus HA-FKBP8 or HA-SELPLG (0 or $400 \mathrm{ng}$ ) as indicated. A Renilla luciferase reporter was included in all transfection mixes and employed for normalization. The relative luciferase activity (fold by luciferase value) was calculated by dividing each normalized average luciferase value by the normalized average mock luciferase value. The data are expressed as the means \pm standard deviation $(n=4)$. FKBP8, FK506-binding protein 8; SELPLG, selectin P ligand; PRL, phosphatase of regenerating liver.

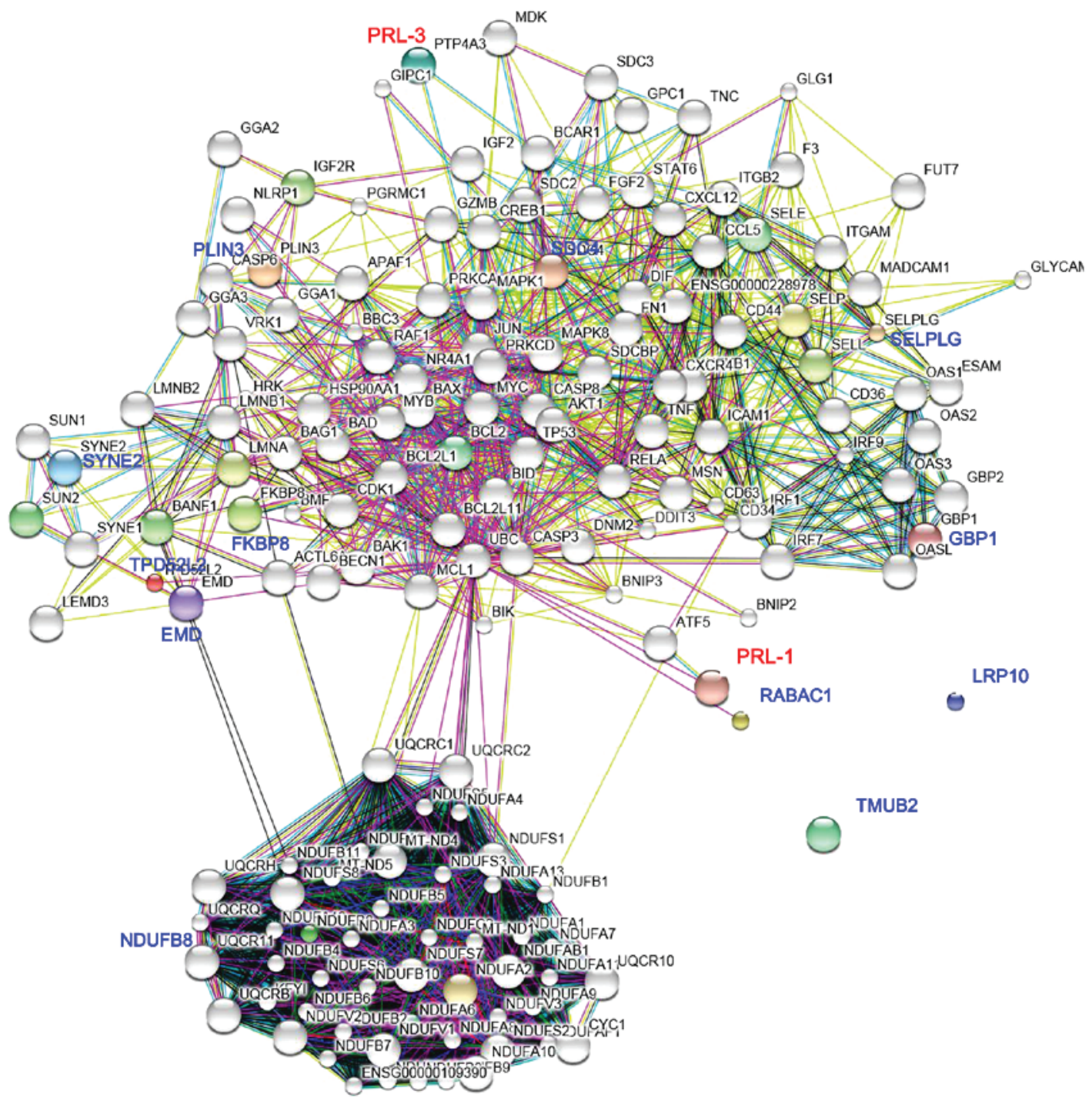

Figure 4. PRL-1, PRL-3 and prey proteins network. The PRL-1, PRL-3-to-cellular protein interaction network was constructed based on the screened proteins in this study using the Search Tool for the Retrieval of Interacting Genes/Proteins database. PRL, phosphatase of regenerating liver. 


\section{Acknowledgements}

This study was supported by the National Research Foundation of Korea (NRF) grant funded by the Korean Government (2013-R1A1A1007596).

\section{References}

1. Al-Aidaroos AQ and Zeng Q: PRL-3 phosphatase and cancer metastasis. J Cell Biochem 111: 1087-1098, 2010.

2. Achiwa H and Lazo JS: PRL-1 tyrosine phosphatase regulates c-Src levels, adherence, and invasion in human lung cancer cells. Cancer Res 67: 643-650, 2007.

3. Stephens BJ, Han H, Gokhale V and Von Hoff DD: PRL phosphatases as potential molecular targets in cancer. Mol Cancer Ther 4: 1653-1661, 2005.

4. Saha S, Bardelli A, Buckhaults P, Velculescu VE, Rago C, St Croix B, Romans KE, Choti MA, Lengauer C, Kinzler KW and Vogelstein B: A phosphatase associated with metastasis of colorectal cancer. Science 294: 1343-1346, 2001.

5. Wang Q, Holmes DI, Powell SM, Lu QL and Waxman J: Analysis of stromal-epithelial interactions in prostate cancer identifies PTPCAAX2 as a potential oncogene. Cancer Lett 175: 63-69, 2002.

6. Parker BS, Argani P, Cook BP, Liangfeng H, Chartrand SD, Zhang M, Saha S, Bardelli A, Jiang Y, St Martin TB, et al Alterations in vascular gene expression in invasive breast carcinoma. Cancer Res 64: 7857-7866, 2004.

7. Miskad UA, Semba S, Kato H and Yokozaki H: Expression of PRL-3 phosphatase in human gastric carcinomas: Close correlation with invasion and metastasis. Pathobiology 71: 176-184, 2004.

8. Wu X, Zeng H, Zhang X, Zhao Y, Sha H, Ge X, Zhang M, Gao X and Xu Q: Phosphatase of regenerating liver-3 promotes motility and metastasis of mouse melanoma cells. Am J Pathol 164: 2039-2054, 2004

9. Wang H, Quah SY, Dong JM, Manser E, Tang JP and Zeng Q: PRL-3 down-regulates PTEN expression and signals through PI3K to promote epithelial-mesenchymal transition. Cancer Res 67: 2922-2926, 2007.

10. Kato H, Semba S, Miskad UA, Seo Y, Kasuga M and Yokozaki H: High expression of PRL-3 promotes cancer cell motility and liver metastasis in human colorectal cancer: A predictive molecular marker of metachronous liver and lung metastases. Clin Cancer Res 10: 7318-7328, 2004.

11. Mizuuchi E, Semba S, Kodama Y and Yokozaki H Down-modulation of keratin 8 phosphorylation levels by PRL-3 contributes to colorectal carcinoma progression. Int J Cancer 124: 1802-1810, 2009.

12. Min SH, Kim DM, Heo YS, Kim YI, Kim HM, Kim J, Han YM, Kim IC and Yoo OJ: New p53 target, phosphatase of regenerating liver 1 (PRL-1) downregulates p53. Oncogene 28: 545-554, 2009.

13. Fiordalisi JJ, Keller PJ and Cox AD: PRL tyrosine phosphatases regulate rho family GTPases to promote invasion and motility. Cancer Res 66: 3153-3161, 2006.

14. Werner SR, Lee PA, DeCamp MW, Crowell DN, Randall SK and Crowell PL: Enhanced cell cycle progression and down regulation of p21 (Cip1/Waf1) by PRL tyrosine phosphatases. Cancer Lett 202: 201-211, 2003.

15. Liang F, Liang J, Wang WQ, Sun JP, Udho E and Zhang ZY: PRL3 promotes cell invasion and proliferation by down-regulation of Csk leading to Src activation. J Biol Chem 282: 5413-5419, 2007.

16. Peng L, Jin G, Wang L, Guo J, Meng L and Shou C: Identification of integrin alphal as an interacting protein of protein tyrosine phosphatase PRL-3. Biochem Biophys Res Commun 342: 179-183, 2006.

17. Hinds PW: Too much of a good thing: The Prl-3 in p53's oyster. Mol Cell 30: 260-261, 2008.
18. Basak S, Jacobs SB, Krieg AJ, Pathak N, Zeng Q, Kaldis P, Giaccia AJ and Attardi LD: The metastasis-associated gene Prl-3 is a p53 target involved in cell-cycle regulation. Mol Cell 30: 303-314, 2008

19. Min SH, Kim DM, Heo YS, Kim HM, Kim IC and Yoo OJ: Downregulation of p53 by phosphatase of regenerating liver 3 is mediated by MDM2 and PIRH2. Life Sci 86: 66-72, 2010.

20. Forte E, Orsatti L, Talamo F, Barbato G, De Francesco R and Tomei L: Ezrin is a specific and direct target of protein tyrosine phosphatase PRL-3. Biochim Biophys Acta 1783: 334-344, 2008.

21. Jeong DG, Kim SJ, Kim JH, Son JH, Park MR, Lim SM, Yoon TS and Ryu SE: Trimeric structure of PRL-1 phosphatase reveals an active enzyme conformation and regulation mechanisms. J Mol Biol 345: 401-413, 2005.

22. Li M, Brooks CL, Wu-Baer F, Chen D, Baer R and Gu W: Mono-versus polyubiquitination: Differential control of p53 fate by Mdm2. Science 302: 1972-1975, 2003.

23. Pascaru M, Tanase C, Vacaru AM, Boeti P, Neagu E, Popescu I and Szedlacsek SE: Analysis of molecular determinants of PRL-3. J Cell Mol Med 13: 3141-3150, 2009.

24. Peters CS, Liang X, Li S, Kannan S, Peng Y, Taub R and Diamond RH: ATF-7, a novel bZIP protein, interacts with the PRL-1 protein-tyrosine phosphatase. J Biol Chem 276: 13718-13726, 2001.

25. Si X, Zeng Q, Ng CH, Hong W and Pallen CJ: Interaction of farnesylated PRL-2, a protein-tyrosine phosphatase, with the beta-subunit of geranylgeranyltransferase II. J Biol Chem 276: 32875-32882, 2001.

26. Sun JP, Wang WQ, Yang H, Liu S, Liang F, Fedorov AA, Almo SC and Zhang ZY: Structure and biochemical properties of PRL-1, a phosphatase implicated in cell growth, differentiation, and tumor invasion. Biochemistry 44: 12009-12021, 2005.

27. Bessette DC, Qiu D and Pallen CJ: PRL PTPs: Mediators and markers of cancer progression. Cancer Metastasis Rev 27: 231-252, 2008.

28. Choi MS, Min SH, Jung H, Lee JD, Lee TH, Lee HK and Yoo OJ: The essential role of FKBP38 in regulating phosphatase of regenerating liver 3 (PRL-3) protein stability. Biochem Biophys Res Commun 406: 305-309, 2011

29. Min SH, Lau AW, Lee TH, Inuzuka H, Wei S, Huang P, Shaik S, Lee DY, Finn G, Balastik M, et al: Negative regulation of the stability and tumor suppressor function of Fbw7 by the Pin1 prolyl isomerase. Mol Cell 46: 771-783, 2012

30. Peng L, Xing X, Li W, Qu L, Meng L, Lian S, Jiang B, Wu J and Shou C: PRL-3 promotes the motility, invasion, and metastasis of LoVo colon cancer cells through PRL-3-integrin beta1-ERK1/2 and-MMP2 signaling. Mol Cancer 8: 110, 2009.

31. Kang CB, Feng L, Chia J and Yoon HS: Molecular characterization of FK-506 binding protein 38 and its potential regulatory role on the anti-apoptotic protein Bcl-2. Biochem Biophys Res Commun 337: 30-38, 2005.

32. Bulgakov OV, Eggenschwiler JT, Hong DH, Anderson KV and $\mathrm{Li}$ T: FKBP8 is a negative regulator of mouse sonic hedgehog signaling in neural tissues. Development 131: 2149-2159, 2004.

33. Fong S, Mounkes L, Liu Y, Maibaum M, Alonzo E, Desprez PY, Thor AD, Kashani-Sabet M and Debs RJ: Functional identification of distinct sets of antitumor activities mediated by the FKBP gene family. Proc Natl Acad Sci USA 100: 14253-14258, 2003.

34. Rosner M, Hofer K, Kubista M and Hengstschläger M: Cell size regulation by the human TSC tumor suppressor proteins depends on PI3K and FKBP38. Oncogene 22: 4786-4798, 2003.

35. Bai X, Ma D, Liu A, Shen X, Wang QJ, Liu Y and Jiang Y: Rheb activates mTOR by antagonizing its endogenous inhibitor, FKBP38. Science 318: 977-980, 2007.

36. Luan SL, Boulanger E, Ye H, Chanudet E, Johnson N, Hamoudi RA, Bacon CM, Liu H, Huang Y, Said J, et al: Primary effusion lymphoma: Genomic profiling revealed amplification of SELPLG and CORO1C encoding for proteins important for cell migration. J Pathol 222: 166-179, 2010. 